\title{
A comparison of four weight estimation systems for paediatric resuscitation
}

\author{
MJ Manyoni, LN Goldstein, M Wells \\ Division of Emergency Medicine, Faculty of Health Sciences, University of the Witwatersrand
}

Corresponding author: Dr Lara Nicole Goldstein (drg666@gmail.com)

\begin{abstract}
Background: One of the most important parameters that must be obtained when resuscitating a critically ill or injured paediatric patient is their weight. The best known paediatric weight estimation system is arguably the Broselow Tape, but the tape has been shown to be very inaccurate. The aim of this study was to determine and compare the accuracy of the Broselow tape, a modified Broselow tape system, the PAWPER XL tape and the hanging leg weight technique for potential utilisation in the paediatric resuscitation setting.

Methods: A convenience sample of 200 children between the ages of 1 month and 16 years were enrolled. All the children's weights were estimated using the Broselow tape, a habitus-modified Broselow tape system, the PAWPER XL tape and the hanging leg weight technique. Overall accuracy was evaluated using the percentage of weight estimations falling within $10 \%$ of actual weight (PW10).

Results: The PAWPER XL tape performed the best, whilst the hanging leg weight technique performed the poorest with PW10s of $74 \%$ and $19.5 \%$, respectively. The Broselow tape with and without habitus-modification only showed modest accuracy, with PW10s of $61.7 \%$ and $59.1 \%$ respectively.

Conclusions: The PAWPER XL tape performed significantly better than other weight estimation systems and is most appropriate for use in South African paediatric emergencies. The habitus-modified Broselow system produced only modest improvement in overall weight estimation accuracy of the Broselow tape.
\end{abstract}

\section{Introduction}

One of the most important parameters that must be obtained when resuscitating a critically ill or injured paediatric patient is their weight. The dose of emergency medications, the volume of intravenous fluids such as in burns fluid replacement or the amount of blood and blood products to be infused in the hypotensive paediatric trauma victim as well as the equipment to be utilised are all determined by the child's weight. ${ }^{1}$ Ideally, a calibrated scale would be used to obtain an accurate weight, but during resuscitation this would be difficult as patients may be immobilised on spine boards or undergoing emergency interventions such as intubation. ${ }^{2}$ Therefore, healthcare providers need a way to rapidly and accurately estimate a child's weight without impeding or causing delays in resuscitation.

There are numerous ways that have been devised to estimate a paediatric patient's weight such as guesses, agebased formulae and length-based or dual length- and habitus- based methods. The American Heart Association recommends that, in the absence of measured weight, length-based weight estimations be used. ${ }^{3}$ The prototype of length-based weight estimation is the Broselow tape, which, apart from the estimated weight, also provides pre-calculated drug dosages and equipment size recommendations. The major drawback of the Broselow tape is that its drug dosages are based on ideal body weight and not a child's actual weight. Therefore, there are inherent risks of under- and over-dosing medications in patients with higher or lower than average weight-for-length, respectively. Body habitus needs to be considered as weight based on length alone has the potential to result in substantial drug dosing errors. ${ }^{4}$ In order to limit these effects in obese children, the manufacturers of the Broselow tape have suggested the use of a visual estimate to allow the examiner to take the body habitus into account and "bump (the child's weight) up" a colour zone for weight estimation and drug dosing purposes. ${ }^{5}$ The accuracy of this technique has only been evaluated once before. ${ }^{6}$ 
As a result of the limitations of length-based weight estimation, Wells et al. developed a dual length- and habitusbased weight estimation tool, the PAWPER (Paediatric Advanced Weight Prediction in the Emergency Room) tape, which takes body habitus into account when estimating weight. It allows the examiner to adjust the weight estimation up or down depending on the child's habitus. It has performed better than the Broselow tape in multiple previous studies. ${ }^{2,4,7}$ The PAWPER tape did not perform as well in a recent study in a very obese population, however, and failed to achieve the same accuracy as in studies in the rest of the world. ${ }^{7}$ As a result, the PAWPER XL tape was developed, which has 7 habitus scores instead of 5 and the length of the tape was extended from $153 \mathrm{~cm}$ to $180 \mathrm{~cm}^{8}$

The hanging leg weight technique was first described in 1990 and was one of the most accurate weight estimations ever reported in its initial study, but no subsequent validation study has been done to assess its accuracy. It is performed on supine children whose fully extended lower limbs are suspended by the heels in a sling hung from a calibrated scale. It uses this weight to predict the actual total body weight from a formula: Estimated weight $=5.176 \times$ Leg Weight $(\mathrm{kg})+3.487 .{ }^{9}$

It is essential that the estimated weight obtained during resuscitation is an accurate weight. The purpose of this study was to determine and compare the accuracy of four paediatric weight estimation modalities in the South African emergency setting: the Broselow tape, a modified Broselow method, the PAWPER XL tape and the hanging leg weight system.

\section{Methods}

\section{Study setting}

Our study was conducted in the Chris Hani Baragwanath Academic Hospital paediatric emergency department in Soweto, Johannesburg, South Africa. It is a tertiary institution, which serves a community of low to middle socioeconomic healthcare users.

\section{Study participants}

A convenience sample of 200 participants between the ages of 1 month and 16 years were enrolled between the period 1 November 2016 and 31 March 2017. This number was predicted to be sufficient and able to detect an absolute difference in weight estimation accuracy of $10 \%$ between weight estimation systems, with a power of $80 \%$ and $95 \%$ confidence. Children that required emergency treatment were excluded. Approval to conduct this study was obtained from the Human Research Ethics Committee of the University of the Witwatersrand (M160631). All parents signed informed consent and assent was obtained from children over the age of seven years.

\section{Study protocol}

Data was obtained and recorded by a doctor who had received training on the use of the PAWPER XL tape, the Broselow tape and the hanging leg weight technique. The data collection procedure was the same for each child. The child, accompanied by the guardian, was ushered into an examination room. The child was undressed to his or her underwear and their habitus was estimated with the aid of reference images (Figure 1) and recorded. The child was then positioned supine on an examination bed. The Broselow tape was used to estimate weight using its standard methodology (described in Table 1). ${ }^{10}$ Children who were considered to be obese, based on a simple visual assessment of habitus, were "bumped up" a colour zone and this weight was recorded as their modified Broselow tape estimated weight. ${ }^{5}$

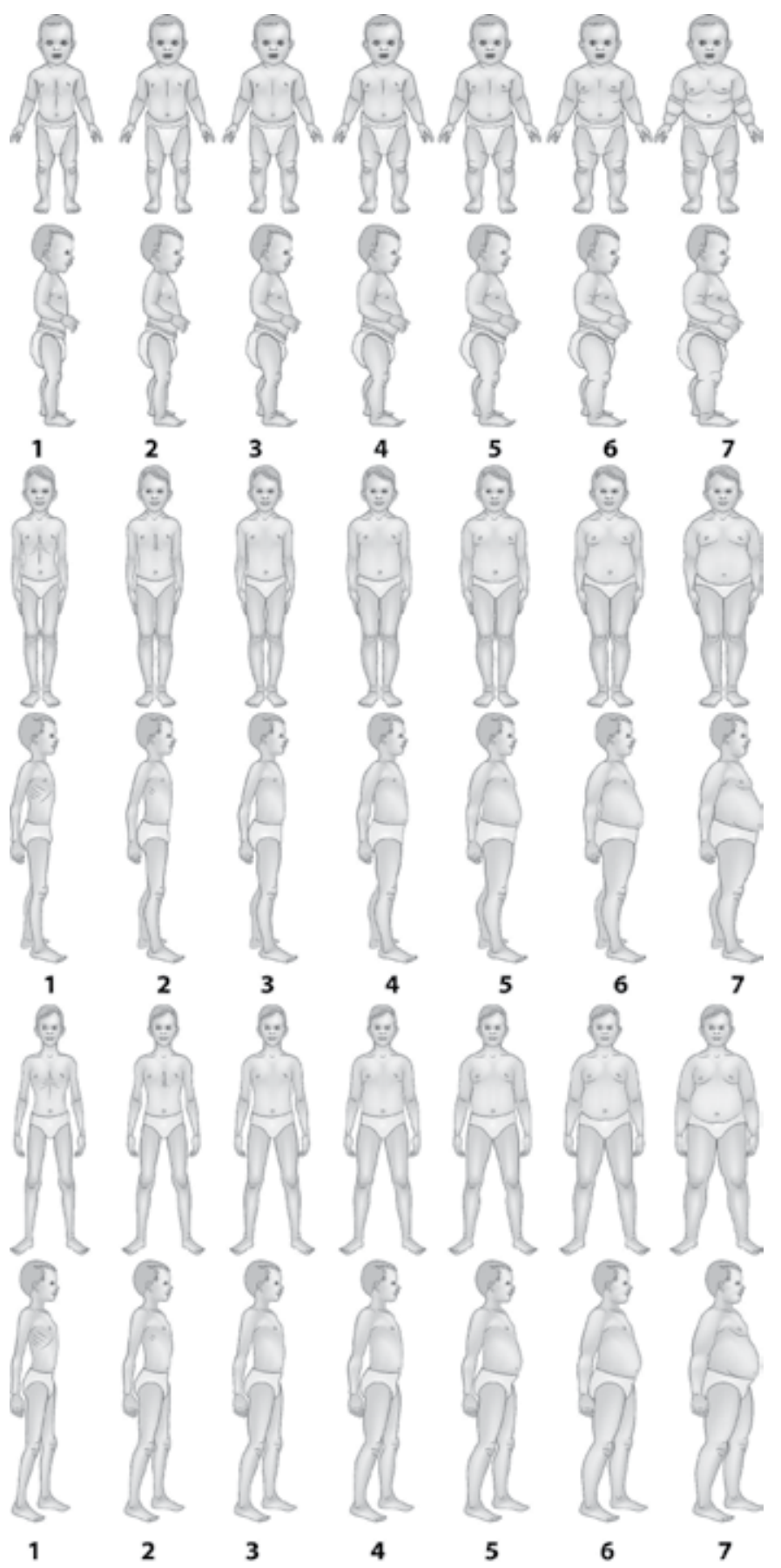

Figure 1. Visual aid used to estimate body habitus. Image courtesy of Professor Mike Wells. 
Table 1. Procedure for performing each weight estimation technique

\section{Broselow tape}

Lay the tape alongside the supine child, with the end that says, "MEASURE FROM THIS END" aligned with the top of the child's head. Then stretch the tape along the length of the child's body and take note of the point at which the child's heel crosses the tape. Read the estimated weight directly off the tape at this length.

\section{Modified Broselow tape technique}

Use the same measurement technique as described above. Then assess the child's habitus using the figural reference images (see Figure 1). If the child is obese (a habitus score of 5 or higher) then adjust the child's estimated weight up a colour zone (e.g. if an obese child falls into the $8 \mathrm{~kg}$ section of the purple zone, the weight will be adjusted up to the $10 \mathrm{~kg}$ section of the purple zone).

\section{PAWPER XL tape}

Firstly, identify the beginning of the tape that bears the label, "Measure from this line". Hold the tape in one hand and line up the beginning of the tape with the most superior part of the child's head. Then stretch the tape in a straight line down the side of the child's body and take note of the length-segment where the heel crosses the tape. This segment represents the ideal body weight segment for that child. Then assign a habitus score to the child. From the figural reference images (see Figure 1), select the image that most closely resembles the habitus of the child that is being assessed. This will provide the most appropriate habitus score. The habitus-adjusted total body weight can then be read off the tape in the appropriate length segment.

\section{Hanging-leg weight technique}

The supine child's legs are supported by the heels in a sling, which is attached to a digital scale. The legs are elevated to 45 degrees and the weight measurement is recorded. This weight is then used in the formula published by the developers of the technique to obtain an estimated weight. The formula is: Estimated weight $=5.176 \mathrm{x}$ leg weight $(\mathrm{kg})+3.487^{9}$

Habitus Score (HS) 1 represents an underweight child, HS3 represents the normal weight child and HS7 represents the severely obese child. The other habitus scores are for the "inbetweeners". Each HS has its own predicted weight in each length segment of the PAWPER XL tape.

Their weight was next estimated using the standard PAWPER XL tape using the PAWPER methodology (described in Table 1). ${ }^{2}$ The children's legs were then put in a sling and weighed; this was recorded as the predicted weight for the hanging leg weight technique using a Hazlo digital luggage scale (described in Table 1). ${ }^{9}$ This scale is accurate for weights between $10 \mathrm{~g}$ and $50 \mathrm{~kg}$. It was calibrated according to the manufacturer's recommendations. Lastly, the children were put on a calibrated scale (SECA 769 COLUMN SCALE) to get their actual weight, which was documented and used as the reference standard.

\section{Data analysis}

Basic demographic data was collected. Children were categorised for subgroup-analysis based on their weight-status and age. The weight categories were based on the habitus score (HS): HS3 represented children of average weight, greater than HS3 represented overweight and less than HS3 represented underweight children. These are the same HS shown in Figure 1. Age-categories of children $\leq 5$ years, children aged 5 to 10 years and children $>10$ years of age were used for subgroup analysis.

The results from the four weight estimation tools were compared to the actual weight (reference standard) using methods that analysed the bias, precision and overall accuracy of the systems. Bias was measured using the mean percentage error (MPE) of the weight estimates. Precision was measured using the Bland \& Altman limits of agreement of the MPE and the root-mean-square percentage error (RMSPE). The overall accuracy was represented by the percentage of weight estimates falling with $10 \%$ and $20 \%$ of actual weight (PW10 and PW20 respectively). Estimated weights greater than $20 \%$ above or below actual weight were deemed to be critical errors. The McNemar test was used to compare the accuracy between each weight estimation tool.

\section{Acceptable outcome criteria}

A weight estimation system was considered to have performed with acceptable accuracy if it achieved a PW10 $>70 \%$ and PW20 > 95\%?

\section{Results}

\section{Demographic information}

There were 200 children enrolled in the study: 113 males $(56.5 \%)$ and 87 females (43.5\%). The median age was 3.0 years (IQR $1.5-6.0)$. The median actual measured weight was $13.4 \mathrm{~kg}$ (IQR 8.7 - 19.7). There were 123 children with an average body habitus (HS3) (61.5\%), 42 underweight children $(21.0 \%)$ and 35 overweight children (17.5\%).

\section{Accuracy of the weight estimation tools}

The Broselow tape could not provide a weight estimate in 7 children $(3.5 \%)$, as they were too tall for the tape.

The PAWPER XL tape was the only weight estimation tool that achieved acceptable accuracy overall, as shown in Table 2. The Broselow tape and the modified Broselow tape techniques achieved intermediate accuracy. The hanging leg weight technique performed poorly in every analysis. Table 3 shows the comparison of the accuracy of the estimation tools using the McNemar test. 
Table 3. The overall statistical test outcomes between the weight estimation tools using the McNemar test

\begin{tabular}{lcc}
\hline & PW10 & PW20 \\
\hline BT vs BT_mod & 0.6828 & 1 \\
BT vs PTXL & 0.0021 & 0.0037 \\
BT vs HLW & $<0.0001$ & $<0.0001$ \\
BT_mod vs PTXL & 0.0101 & 0.006 \\
BT_mod vs HLW & $<0.0001$ & $<0.0001$ \\
PTXL vs HLW & $<0.0001$ & $<0.0001$
\end{tabular}

$\mathrm{BT}=$ Broselow Tape $; \mathrm{BT} \_$mod $=$Broselow tape with modified habitus; PTXL = PAWPER XL Tape; HLW = Hanging leg weight technique

\section{Weight category}

In each weight category, the PAWPER XL tape showed the lowest bias, narrowest limits of agreement and the greatest accuracy of the weight estimation tools. Its poorest performance was noted in underweight children, with a PW10 and PW20 of $71.4 \%$ and $90.5 \%$, respectively. The performance of the weight estimation tools is illustrated in Figure 2.

\section{Age category}

The PAWPER XL tape performed the best in all three age categories as illustrated in Figure 3. In children older than 10 years of age, the Habitus Modified Broselow Tape had the lowest bias (-1.8) and predicted weight accurately with $73.7 \%$ and $89.5 \%$ of estimations within $10 \%$ and $20 \%$ of actual weight, respectively. However, the PAWPER XL tape was able to predict all of the participants' weights within $20 \%$ of actual weight and $84.6 \%$ within $10 \%$ of their actual weight.

Figure 4 illustrates the percentage of critical errors (weight estimation error $>20 \%$ ) produced by each estimation tool.

\section{Discussion}

\section{Study sample demographics}

In this study from a low to middle socioeconomic population, there was a high prevalence of average weight children followed by underweight children. This is a similar distribution noted by Georgoulas et al. whose study was also based in a public hospital serving a low to middle socioeconomic class in South Africa. Moreover, Shisana et al. found that $13.5 \%$ of South African children are overweight, which is in keeping with our $17.5 \%$, making our sample suitable and probably generalisable to the area and similar populations in South Africa. ${ }^{11}$

\section{Performance of the PAWPER XL tape}

The PAWPER tape and PAWPER XL tape were originally developed and validated in South Africa, the latter being the most accurate weight estimation tool in this study. It achieved the acceptable outcome criteria overall, having good accuracy 


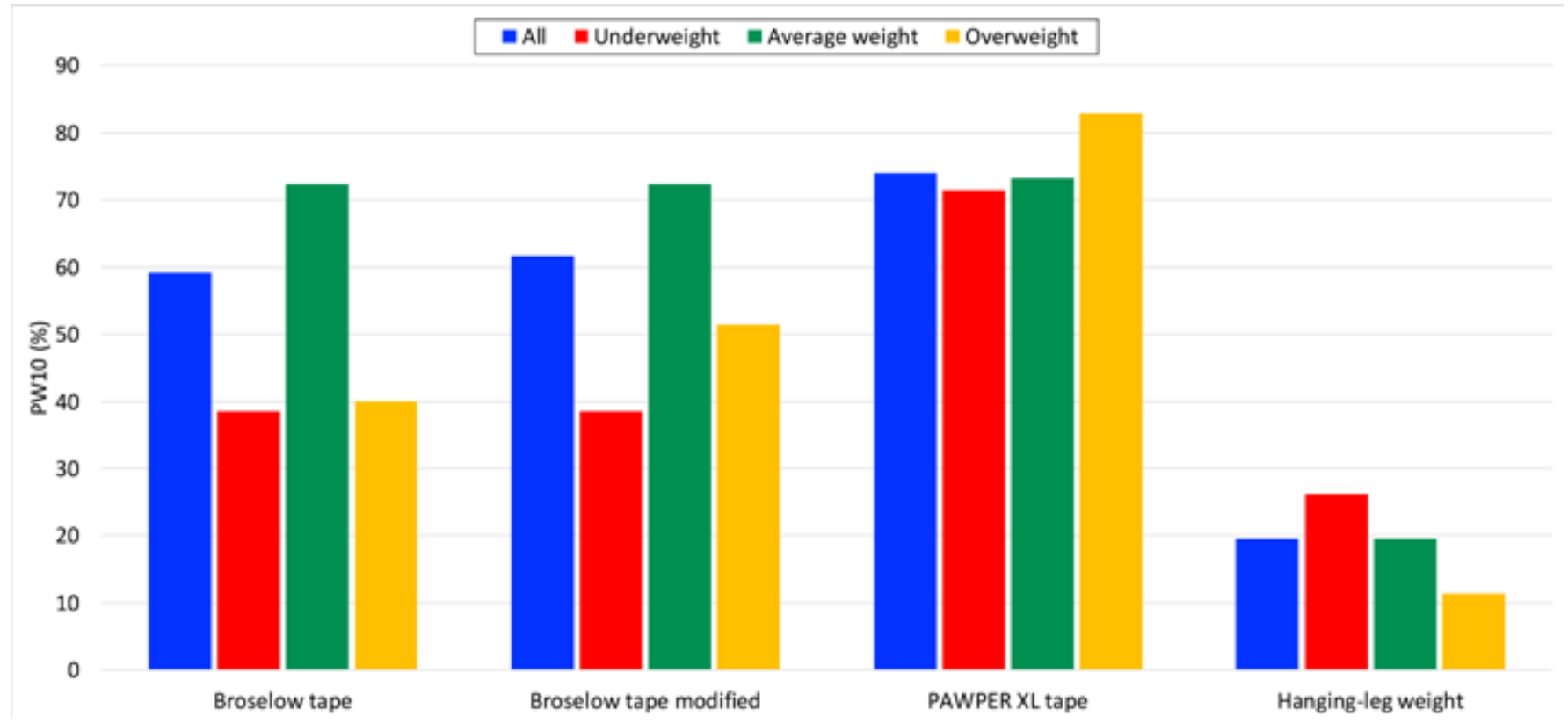

Figure 2. The accuracy of weight estimation tools to estimate weight within $10 \%$ of actual weight in the weight subgroups

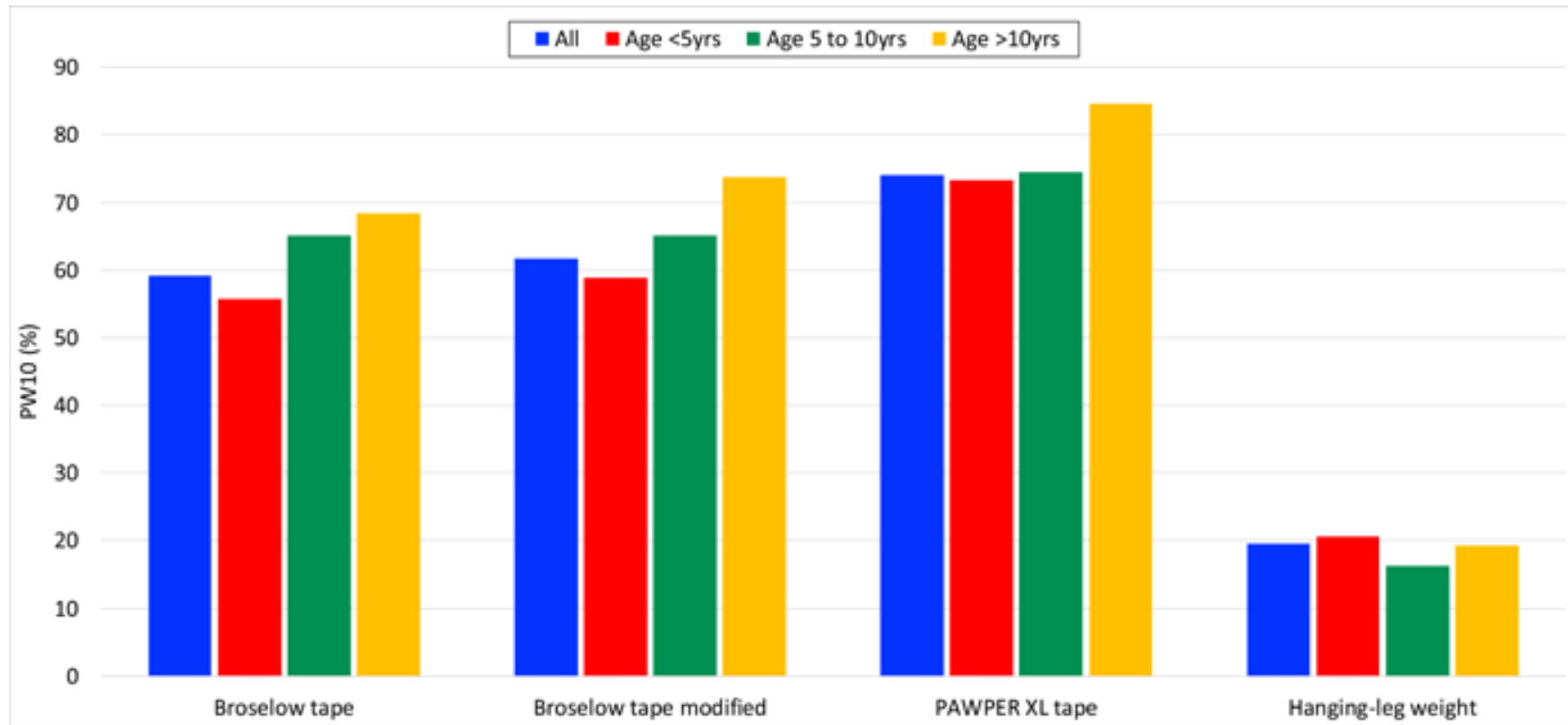

Figure 3. The accuracy of weight estimation tools to predict weight within $10 \%$ of actual weight in each age category

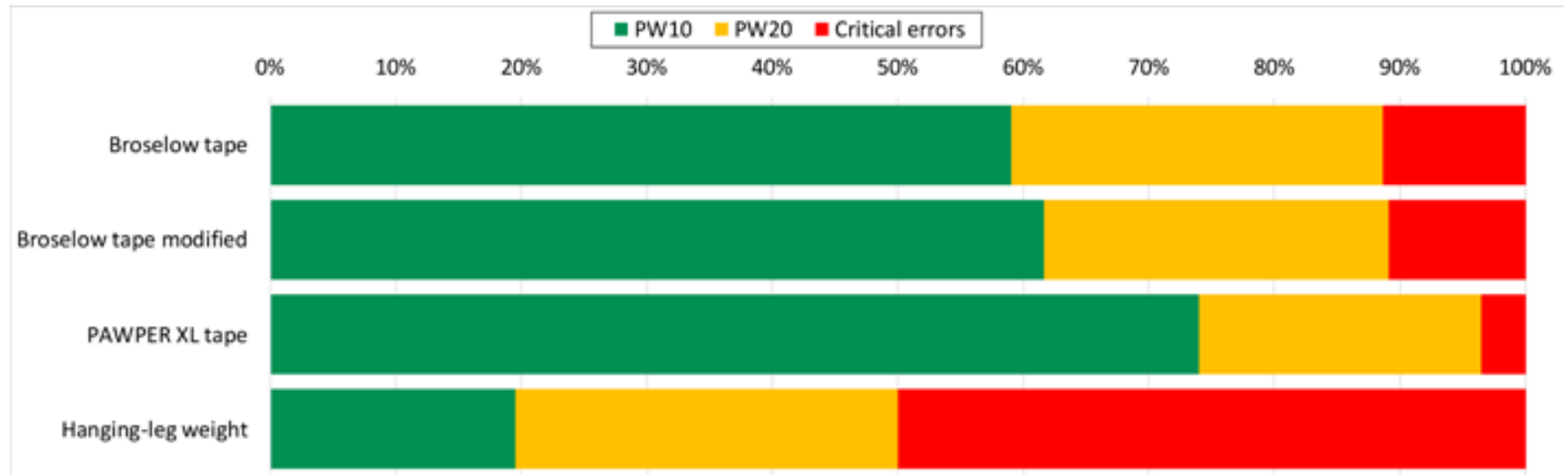

Figure 4. The overall percentage of critical errors made by each weight estimation tool 
in the whole group and every subgroup (see Table 2). It was the most consistent and reproducible tool, with virtually neutral bias (MPE 0.2) and minimal differences between age groups and habitus types. Its poorest performance was in the underweight habitus type, producing a PW20 that did not meet our acceptable accuracy of $95 \%$.

Taking into account its poorest performance, the PAWPER XL tape still performed significantly better than the other weight estimation tools, confirming that length-based estimation tools with body habitus modifications are the most accurate..$^{4,7}$

\section{Performance of the Broselow tape and a modified Broselow tape system}

The Broselow tape is probably the best-known and most widely utilised paediatric weight estimation tool. It has been in clinical use for more than 30 years. Throughout the years and across the world, it has shown to have poor accuracy in estimating weight, especially in children with extremes of habitus. ${ }^{12,13}$ In an attempt to improve the accuracy of the Broselow tape, the manufacturers have suggested that a visual estimate of body habitus can be used to modify the estimate of body weight in overweight or obese children. ${ }^{5}$ Overall, in this study, the unmodified Broselow tape did not meet the acceptable accuracy of $95 \%$ and the habitus modification thereof improved its PW10 and PW20 by only $2.6 \%$ and $0.5 \%$ respectively. In overweight children specifically, it improved the PW10 and PW20 by $11.4 \%$ and $2.8 \%$, respectively. Furthermore, with the incorporation of the body habitus, the tendency to underestimate obese children decreased from a MPE of -10.4 to -8.3 . This is in keeping with numerous studies done in South Africa and internationally showing the tape's inaccuracy in children at extremes of weights. ${ }^{6,12,13}$ Despite these improvements, the habitus modified Broselow Tape only performed with acceptable accuracy in the average weight subgroup but failed to reproduce this in all the other categories. Its worst performance was seen in the underweight subgroup, with a significantly large bias to overestimate their weight. Clark et al. found the same in a study that took place in a low socio-economic population in Sudan. ${ }^{12}$ In the South African population, the Broselow tape and habitus modified Broselow tape system only met acceptable weight estimation criteria in the average weight subgroup and failed in all the other categories.

\section{Performance of the hanging leg weight technique}

In its original study, the hanging leg weight technique was performed on unmoving, anaesthetised children and was able to achieve a PW10 of $73.7 \% .{ }^{9}$ In our study, this could not be reproduced.

This system was very inaccurate across the whole group as well as in each subgroup. It had a large bias to underestimate weight (MPE -6.5) overall, but overestimated weight in underweight children. The hanging leg weight technique performed significantly worse than all the other tools and is not suitable for a South African population. The technique's shortcomings are predictable when compared to the original study, in that our participants were not inert. It was virtually impossible to weigh a fully conscious child's legs without them moving and affecting the measurement. Moreover it assumes that leg weight and body weight are related independent of body habitus, length and age. ${ }^{9}$ However, studies since the originally published hanging leg weight technique have showed that body weight is intimately related to length and more importantly body habitus. ${ }^{3-5,7}$ The hanging leg weight technique is not suitable for clinical use. In our experience, this technique was not user-friendly and is impractical.

\section{Critical weight estimation errors}

The corollary to a tape performing with acceptable accuracy $(\mathrm{PW} 10>70 \%$, PW20 $>95 \%)$ is its generation of critical errors. $^{7}$ It was not surprising that the hanging leg weight technique had the highest critical error rate of $50 \%$. The Broselow Tape, modified Broselow tape method and PAWPER XL tape critical error rates were $11.4 \%, 10.9 \%$ and $3.5 \%$, respectively (Figure 4 ). O'Leary et al. found similar results in an ethnically diverse Australian population, with the Broselow tape having a critical error rate of $12.5 \%$ and the PAWPER tape maintaining its $3.5 \%$ rate. ${ }^{4}$ Similar critical errors have been found in studies done in the South African setting as well. ${ }^{14}$

\section{Difficulties experienced with the tools}

The Broselow tape was too short for seven of the participants, resulting in weights that could not be estimated. This is a welldocumented limitation and disadvantage of the tape, not only in South Africa, but other countries too. ${ }^{15}$ The tape is limited between the lengths of 46 to $143 \mathrm{~cm}$ whilst the PAWPER XL tape has an upper limit of $180 \mathrm{~cm}$. The PAWPER XL and hanging leg weight technique could be used on all the children, although the latter with some difficulty.

\section{Utility of weight estimation tools in resuscitation}

Accurate weight prediction of paediatric patients is crucial to their safe and effective management in the emergency setting. The weight estimation system utilised for this purpose needs to be readily available, easy-to-use and accurate. The PAWPER XL tape was the most accurate and is substantially cheaper in our setting than the other methods analysed. ${ }^{2,8,14}$

\section{Limitations}

Our study was done in South Africa, Soweto, a low to middle socioeconomic class of people. One can appreciate the potential drawback of using the Broselow tape in such a population, since the tape was developed from North American paediatric growth charts.

One of the major limitations to the study is that it was done in one hospital and may not be generalisable to other populations. The number of participants was also relatively small. Further research on a larger scale is required especially with a greater representation of obese and underweight children.

A single researcher (MM) took measurements and therefore 
interobserver variability of the methods could not be assessed.

\section{Conclusion}

In this study population the PAWPER XL tape was the most accurate weight estimation tool. It performed better than the other weight estimation tools in all categories and met acceptable accuracy in all categories but underweight children. The Broselow tape and a habitus modified Broselow tape system had similar performance but the modified system was understandably slightly more accurate in overweight children. Acceptable accuracy was obtained in the average weight population only. The hanging leg weight technique was grossly inaccurate and is not recommended for clinical use.

In South African paediatric emergencies, the PAWPER XL tape is the most accurate tool for weight estimation.

\section{References}

1. Khouli M, Ortiz MI, Romo-Hernández G, Martínez-Licona D, Stelzner SM. Use of the Broselow tape in a Mexican emergency department. J Emerg Med. Jun 2015;48(6):660-6. doi: 10.1016/j.jemermed.2014.12.082

2. Wells M, Coovadia A, Kramer E, Goldstein L. The PAWPER tape: A new concept tape-based device that increases the accuracy of weight estimation in children through the inclusion of a modifier based on body habitus. Resuscitation. Feb 2013;84(2):227-32. doi: 10.1016/j.resuscitation.2012.05.028

3. Kleinman ME, Chameides L, Schexnayder SM, Samson RA, Hazinski MF, Atkins DL, et al. Part 14: pediatric advanced life support: 2010 American Heart Association Guidelines for Cardiopulmonary Resuscitation and Emergency Cardiovascular Care. Circulation. 2 Nov 2010;122(18 Suppl 3):S876-908. doi: 10.1161/CIRCULATIONAHA.110.971101

4. O'Leary F, John-Denny B, McGarvey K, Hann A, Pegiazoglou I, Peat J. Estimating the weight of ethnically diverse children attending an Australian emergency department: a prospective, blinded, comparison of age-based and length-based tools including Mercy, PAWPER and Broselow. Arch Dis Child. Jan 2017;102(1):46-52. doi: 10.1136/archdischild-2016-310917
5. Deboer S, Seaver M, Broselow J. Color coding to reduce errors. Am J Nurs. Aug 2005;105(8):68-71. PMID: 16062063

6. Wells M, Goldstein L, Bentley A. The accuracy of weight estimation by the Broselow tape can be substantially improved by including a modifier based on a visual assessment of body habitus. Pediatr Res. 18 Oct 2017. doi: 10.1038/pr.2017.222

7. Wells M, Goldstein L, Bentley A. The accuracy of emergency weight estimation systems in children - a systematic review and meta-analysis. Int J Emerg Med. 2017;10:29. doi: 10.1186/ s12245-017-0156-5

8. Wells M, Goldstein L, Bentley A. A validation study of the PAWPER XL tape: accurate estimation of both total and ideal body weight in children up to 16 years of age. Trauma Emerg Care. 2017;2(4):1-8. doi: 10.15761/TEC.1000141

9. Haftel AJ, Khan N, Lev R, Schonfeld N. Hanging leg weight a rapid technique for estimating total body weight in pediatric resuscitation. Ann Emerg Med. May 1990;19(5):523-6. PMID: 2331096

10. Frush K. Study packet for the correct use of the Broselow ${ }^{\mathrm{TM}}$ Pediatric Emergency Tape. Durham, NC: Duke University Medical Center. Accessed 5 May 2018. Available from: http:// www.progress.com.sg/wp-content/uploads/2015/06/DEPS_ Broselow_Study.pdf

11. Shisana O, Labadarios D, Rehle T, Simbayi L, Zuma K, Dhansay A, et al. \& SANHANES-1 Team (2013) South African National Health and Nutrition Examination Survey (SANHANES-1). Cape Town: HSRC Press.

12. Clark MC, Lewis RJ, Fleischman RJ, Ogunniyi AA, Patel DS, Donaldson RI. Accuracy of the Broselow Tape in South Sudan, "The Hungriest Place on Earth". Acad Emerg Med. Jan 2016;23(1):21-8. doi: 10.1111/acem.12854

13. Geduld H, Hodkinson PW, Wallis LA. Validation of weight estimation by age and length based methods in the Western Cape, South Africa population. Emerg Med J. Oct 2011;28(10):85660. doi: 10.1136/emj.2010.098640

14. Wells M, Goldstein LN, Bentley A. Development and validation of a method to estimate body weight in critically ill children using length and mid-arm circumference measurements: The PAWPER XL-MAC system. S Afr Med J. 31 Oct 2017;107(11):1015-21. doi: 10.7196/SAMJ.2017.v107i11

15. Cattermole GN, Leung PY, Graham CA, Rainer TH. Too tall for the tape: the weight of schoolchildren who do not fit the Broselow tape. Emerg Med J. Jul 2014;31(7):541-4. doi: 10.1136/emermed-2012-202325 